\title{
A Systematic Impedance Model for Non-Linear Helmholtz Resonator Liner
}

\author{
Deepesh Kumar Singh* and Sjoerd W. Rienstra ${ }^{\dagger}$ \\ Department of Mathematics and Computer Science, TU Eindhoven, The Netherlands
}

\begin{abstract}
The usual non-linear corrections for a Helmholtz resonator type impedance do not seem to be based on a systematic asymptotic solution of the pertaining equations. We aim to present a systematic derivation of a solution of the non-linear Helmholtz resonator equation, in order to obtain analytically expressions for impedances close to resonance, while including non-linear effects. The amplitude regime considered is such that when we stay away from the resonance condition, the non-linear terms are relatively small and the solution obtained is of the linear equation (formed after neglecting the non-linear terms). Close to the resonance frequency, the non-linear terms can no longer be neglected and algebraic equations are obtained that describe the corresponding non-linear impedance.
\end{abstract}

\section{Introduction}

An important type of acoustic liner constitutes of a honeycomb array of small cells called Helmholtz resonators. The Helmholtz resonator is a cavity filled with air and having a small opening called the neck. When excited with a fluctuating external pressure, the mass of air plug inside the neck moves against the large volume of compressible air inside the cavity, which acts as a spring, while viscous forces and vortex shedding generate dissipation of energy. Altogether this establishes a mass-spring-damper system. The damping is normally relatively small such that a resonance frequency can be identified. At and near resonance, the dissipation is largest and so narrow band sound absorption is achieved for frequencies close to resonance. This process is the basic design criterion for the liners. The resonator, as "seen" from outside, is characterized by its impedance $Z=Z(\omega)$, relating (spatially averaged) pressure and velocity at the wall. Ideally, $Z$ is a wall property and independent of the acoustic field. However, in particular near resonance $Z$ is amplitude dependent for high but relevant amplitudes, for example of the "buzz saw" noise in a turbofan engine due to the shocks produced in front of the fan at take off and the blade tips operates in a supersonic regime [1,2]. Since liners are designed to operate at resonance, it is important to know quantitatively and understand qualitatively such impedances $Z$ with good precision.

The non-linear effects are mainly of hydrodynamical origin, due to vortex shedding at outflow from the opening. This is physically a rather complicated process [3-7] and the existing non-linear corrections of the impedance are either based on physically inspired modelling assumptions that do not aim to solve the equations of the non-linear resonator [8-12], or by fully CFD or DNS simulations [1, 13-18]. Only Innes and Crighton [19] analysed a non-linear Helmholtz resonator equation systematically, but their configuration is different from ours. They considered very high amplitudes with grazing flow and thus a (slightly) different differential equation.

The extra complication of grazing flow along the liner wall will not be considered here. It is important if the mean flow boundary layer is thin enough and the resonator outflow velocity is high but not very

\footnotetext{
${ }^{*}$ Doctoral candidate, Dept. Math. \& Comp. Sc., Eindhoven University of Technology, Netherlands.

${ }^{\dagger}$ Associate Professor, Dept. Math. \& Comp. Sc., Eindhoven University of Technology, Netherlands, Senior Member AIAA. Copyright (c) 2013 by D.K. Singh, S.W. Rienstra. Published by the American Institute of Aeronautics and Astronautics, Inc. with permission.
} 
high. The present work focuses on a systematic derivation of an asymptotic solution of a stand-alone non-linear Helmholtz resonator equation from first principles.

We start with the classical modelling of the Helmholtz resonator and formulate a perturbation problem in terms of a small parameter $\varepsilon$ which is based on the exciting amplitude. The stationary solution of this problem is solved asymptotically up to second order. Secular effects of the external forcing are treated in the usual way by a suitable Lindstedt-Poincaré type transformation. A non-standard problem was the modulus term $|u|$ of the velocity. This prohibits a standard asymptotic expansion because the location of the zero's of $u$ are a priori unknown. This problem has been tackled by adding an unknown shift of the origin, to be determined along the construction of the solution, and using the fact that the stationary solution has the same periodicity as the driving force.

\section{Mathematical Formulation}

A sketch of the Helmholtz resonator considered is shown in Fig.11, A simple and classic model (in various forms presented in the previously mentioned literature), that includes non-linear separation effects for the air flow in and out the neck, is derived as follows. If the cross-sectional area $S_{b}$ of the bottle is

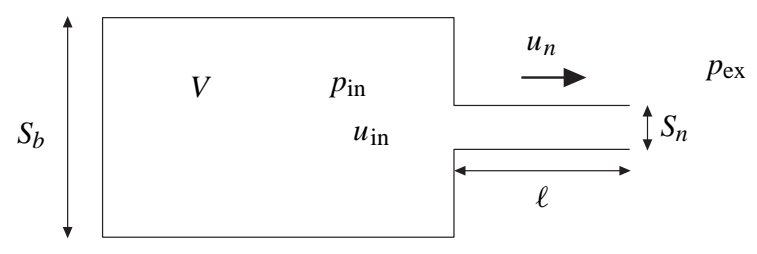

Figure 1. Helmholtz resonator

large compared to the cross sectional area $S_{n}$ of the neck, the acoustic velocities in the bottle will be small compared to those in the neck. Hence we may assume that the pressure and density perturbations $p_{\text {in }}$ and $\rho_{\text {in }}$ in the bottle are uniform. If the cavity neck is acoustically compact, i.e. $k \ell \ll 1$ for a typical wave number $k=\omega / c_{0}$, we can neglect compressibility in the neck and integrate along a cross section $S_{n}$ the line integral of the momentum equation

$$
\rho_{0}\left(\frac{\partial \boldsymbol{v}}{\partial t}+\boldsymbol{v} \cdot \nabla \boldsymbol{v}\right)+\nabla p=\mu \nabla^{2} \boldsymbol{v}
$$

along a streamline from a point inside to a point outside to obtain for the cross sectionally averaged velocity $\bar{v}$ the relation

$$
\rho_{0} \int_{\text {in }}^{\mathrm{ex}} \frac{\partial \overline{\boldsymbol{v}}}{\partial t} \cdot \mathbf{d} \boldsymbol{s}+\frac{1}{2} \rho_{0}\left(u_{\mathrm{ex}}^{2}-u_{\mathrm{in}}^{2}\right)+\left(p_{\mathrm{ex}}-p_{\mathrm{in}}\right)=\int_{\text {in }}^{\mathrm{ex}} \mu \overline{\nabla^{2} \boldsymbol{v}} \cdot \mathbf{d} \boldsymbol{s} .
$$

Assuming that the streamline does not change in time (for example the center streamline) we have

$$
\int_{\text {in }}^{\text {ex }} \frac{\partial \bar{v}}{\partial t} \cdot \mathbf{d} s=\frac{\mathrm{d}}{\mathrm{d} t} \int_{\text {in }}^{\mathrm{ex}} \overline{\boldsymbol{v}} \cdot \mathbf{d} \boldsymbol{s}
$$

The velocity line integral evidently scales on a typical length times a typical velocity. If end effects are minor, we can use the neck flux velocity $u_{n}$ with a corresponding length being the neck length $\ell$, added by a small end correction $\delta$ to take into account the inertia of the acoustic flow at both ends just outside the neck (inside and outside the resonator). Then we have

$$
\int_{\text {in }}^{\text {ex }} \overline{\boldsymbol{v}} \cdot \mathbf{d} s=(\ell+2 \delta) u_{n}
$$


For the stress term line integral we observe that, apart from $u_{n}$ itself, it will depend on flow profile, Reynolds number, wall heat exchange, turbulence, separation from sharp edges, and maybe more. Following Melling [9], we will take these effects together in a resistance factor $R$, which will a priori be assumed to be relatively small, in order to have resonance and a small decay per period.

$$
\int_{\text {in }}^{\text {ex }} \mu \overline{\nabla^{2} v} \cdot \mathbf{d} s=-R u_{n} .
$$

(Note that this form is exact for a Poiseuille flow with parabolic profile). Due to separation from the outer exit, we have with outflow $u_{\text {in }} \simeq 0$ with $u_{\mathrm{ex}}=u_{n}$ jetting out, while similarly during inflow, $u_{\mathrm{ex}} \simeq 0$ with $u_{\text {in }}=u_{n}$ jetting into the cavity. The pressure in the jets, however, has to remain equal to the surrounding pressure ( $p_{\mathrm{ex}}$ and $p_{\text {in }}$ respectively) because the boundary of the jet cannot support a pressure difference. Therefore, we have altogether

$$
\rho_{0}(\ell+2 \delta) \frac{\mathrm{d}}{\mathrm{d} t} u_{n}+\frac{1}{2} \rho_{0} u_{n}\left|u_{n}\right|+R u_{n}=p_{\text {in }}-p_{\text {ex }} .
$$

The second equation between $p_{n}$ and $u_{n}$ is obtained by applying the integral mass conservation law on the volume $V$ of the cavity. The change of mass must be equal to the flux through the cavity neck, which is in linearised form for the density perturbation $\rho_{\text {in }}$

$$
V \frac{\mathrm{d} \rho_{\text {in }}}{\mathrm{d} t}=-\rho u_{n} S_{n} \approx-\rho_{0} u_{n} S_{n} .
$$

Assuming an adiabatic compression of the fluid in the cavity, we have $p_{\text {in }}=c_{0}^{2} \rho_{\text {in }}$. Elimination of $\rho_{\text {in }}$ and $u_{n}$ from (5) by using (6) and redefining $(\ell+2 \delta):=\ell$ yields the non-linear Helmholtz resonator equation

$$
\frac{\ell V}{c_{0}^{2} S_{n}} \frac{\mathrm{d}^{2} p_{\text {in }}}{\mathrm{d} t^{2}}+\frac{V^{2}}{2 \rho_{0} c_{0}^{4} S_{n}^{2}} \frac{\mathrm{d} p_{\text {in }}}{\mathrm{d} t}\left|\frac{\mathrm{d} p_{\text {in }}}{\mathrm{d} t}\right|+\frac{R V}{\rho_{0} c_{0}^{2} S_{n}} \frac{\mathrm{d} p_{\text {in }}}{\mathrm{d} t}+p_{\text {in }}=p_{\text {ex }} .
$$

For a proper analysis it is most clarifying to rewrite the equation into non-dimensional variables. For this we need an inherent timescale and pressure level. For vanishing amplitudes and negligible dissipation the equation describes a harmonic oscillator, so the reciprocal of its angular frequency

$$
\omega_{0}=\frac{c_{0}}{\ell}\left(\frac{\ell S_{n}}{V}\right)^{1 / 2}
$$

is a suitable timescale of the problem. By dividing the non-linear damping term by the acceleration term we find the pressure level $2 \rho_{0} c_{0}^{2} \ell S_{n} / V$ at which the non-linear damping would be just as large as the other terms. So for a pressure that is a small fraction $\varepsilon$ of this level we have a problem with only little non-linear damping. In addition we assume that the linear damping is small and of the same order of magnitude as the non-linear damping (that is to say: near resonance. Away from resonance the nonlinear term will be relatively smaller). Also, the driving amplitude $p_{\text {ex }}$ will be an order smaller than $p_{\text {in }}$. In order to make all this explicit we introduce a small parameter (via the external forcing amplitude), and make dimensionless

$$
\tau=\omega_{0} t, \quad p_{\text {in }}=2 \varepsilon \rho_{0} c_{0}^{2}\left(\frac{\ell S_{n}}{V}\right) y, \quad p_{\mathrm{ex}}=2 \varepsilon^{2} \rho_{0} c_{0}^{2}\left(\frac{\ell S_{n}}{V}\right) F, \quad R=\varepsilon \rho_{0} c_{0}\left(\frac{\ell S_{n}}{V}\right)^{1 / 2} r,
$$

where $0<\varepsilon \ll 1$ and $r, y, F=O(1)$.

Suppose that we excite the Helmholtz resonator harmonically, such that $p_{\mathrm{ex}}=C \cos (\omega t)+H(t)$ consists of a time-harmonic component of frequency $\omega$ plus a small contribution of higher harmonics $H$ due to the interaction with the resonator 1 . In the scaled variables $\tau$ and $F$ this becomes

$$
F=F_{0} \cos (\Omega \tau)+\varepsilon^{v} h(\tau), \quad \Omega=\frac{\omega}{\omega_{0}},
$$

\footnotetext{
${ }^{1} \mathrm{H}$ will play no role in the results, but appears from the liner application of section $\nabla$. Here, the external forcing field is a combination of incident and reflected waves, say $p_{\mathrm{ex}}=f(t)+g(t)$ and $v_{\mathrm{ex}} \propto f(t)-g(t)$. If incident part $f$ is harmonic, reflected part $g$ will be harmonic plus higher harmonics. We will see, however, that these higher harmonics are one or two orders of magnitude smaller, and therefore play no role in $y_{1}$, eq. (31), resp. $Y_{1}$, eq. (16).
} 
where $v=1$ in the resonant case and $v=2$ in the non-resonant case.

Note that $\varepsilon$ is a bookkeeping parameter, meant to measure the "smallness" of the various parameters and variables. In practice it is determined by the external forcing $p_{\mathrm{ex}}$, so in the simple case of a harmonic excitation we can take $F_{0}=1$, and this will be done in any example below.

Finally we arrive at the weakly nonlinear forced oscillator as given by (10). The initial conditions are not important as we are interested only in the stationary state ${ }^{2}$ of the oscillator synchronised with the forcing.

$$
\frac{\mathrm{d}^{2} y}{\mathrm{~d} \tau^{2}}+\varepsilon \frac{\mathrm{d} y}{\mathrm{~d} \tau}\left|\frac{\mathrm{d} y}{\mathrm{~d} \tau}\right|+\varepsilon r \frac{\mathrm{d} y}{\mathrm{~d} \tau}+y=\varepsilon F_{0} \cos (\Omega \tau)+\varepsilon^{1+v} h(\tau) .
$$

We note in passing that the problem considered by Innes and Crighton [19] relates to ours if we replace $y^{\prime}\left|y^{\prime}\right|$ by $y^{\prime}|y|$, assume $y=O\left(\varepsilon^{-2}\right)$ and $F=O\left(\varepsilon^{-4}\right)$, and neglect $r$.

\section{Asymptotic Analysis}

\section{III.A. Non-Resonant Case}

Away from resonance, when $1-\Omega^{2}=O(1)$, the perturbation problem is regular and relatively straightforward. We will include it here for reference.

We look for solutions of

$$
y^{\prime \prime}+\varepsilon y^{\prime}\left|y^{\prime}\right|+\varepsilon r y^{\prime}+y=\varepsilon F_{0} \cos (\Omega \tau)+\varepsilon^{3} h(\tau)
$$

that are only caused by the external forcing. Since this forcing term is $O(\varepsilon)$ and we are not near resonance, the response is of the same order of magnitude, and we transform $y=\varepsilon Y$, where $Y=O(1)$.

$$
Y^{\prime \prime}+\varepsilon^{2} Y^{\prime}\left|Y^{\prime}\right|+\varepsilon r Y^{\prime}+Y=F_{0} \cos (\Omega \tau)+\varepsilon^{2} h(\tau) .
$$

After substituting the assumed expansion $Y(\tau ; \varepsilon)=Y_{0}(\tau)+\varepsilon Y_{1}(\tau)+\varepsilon^{2} Y_{2}(\tau)+\ldots$ and collecting the coefficients of $O(1)$, we have

$$
Y_{0}^{\prime \prime}+Y_{0}=F_{0} \cos (\Omega \tau) .
$$

The solution that follows the driving force is periodic with frequency $\Omega$ and so

$$
Y_{0}=\frac{F_{0}}{1-\Omega^{2}} \cos (\Omega \tau),
$$

Next we collect the coefficients of $O(\varepsilon)$ to obtain

$$
Y_{1}^{\prime \prime}+Y_{1}=-r Y_{0}^{\prime}=\frac{r F_{0} \Omega}{1-\Omega^{2}} \sin (\Omega \tau)
$$

with solution

$$
Y_{1}=\frac{r F_{0} \Omega}{\left(1-\Omega^{2}\right)^{2}} \sin (\Omega \tau) \text {. }
$$

We may go on to $O\left(\varepsilon^{2}\right)$ and find the appearance of higher harmonics. Efficiently collecting terms together, we obtain for the full solution

$$
y=\varepsilon F_{0} \frac{\left(1-\Omega^{2}\right) \cos \Omega t+\varepsilon r \Omega \sin \Omega t}{\left(1-\Omega^{2}\right)^{2}+\varepsilon^{2} r^{2} \Omega^{2}}+O\left(\varepsilon^{3}\right)
$$

showing that the response is indeed $O(\varepsilon)$ and follows the excitation almost in phase $\left(1-\Omega^{2}>0\right)$ or anti-phase $\left(1-\Omega^{2}<0\right)$. This is not the case anymore near resonance when $1-\Omega^{2}=O(\varepsilon)$.

\footnotetext{
${ }^{2}$ In the appendix it is proved that solutions of this equation (10) are stable, so the stationary solution exists.
} 


\section{III.B. Resonant Case}

Near resonance when $1-\Omega^{2}=O(\varepsilon)$, it was assumed and indeed confirmed by (17) that the amplitude $y$ rises to levels of $O(1)$, and the assumption that the non-linear damping is negligible to leading orders is not correct. As the physics of the problem essentially change when $\Omega=1+O(\varepsilon)$, we introduce a parameter $\Delta=O(1)$ and assume that

$$
\Omega=1+\varepsilon \Delta .
$$

However, posed in this form we obtain secular terms in the expansion $\cos (\tau+\varepsilon \Delta \tau)=\cos (\tau)-$ $\varepsilon \Delta \tau \sin (\tau)+\ldots$ of the driving force, which prohibits a uniform approximation of $y$ later [20, sec 15.3.2]. Therefore we remove the $\varepsilon$-dependence from the driving force by absorbing $\Omega$ into a new time coordinate.

Moreover, the asymptotic expansion of the modulus $\left|y^{\prime}\right|$ introduces difficulties near the $\varepsilon$-dependent (and unknown) zero's of $y^{\prime}$. This will be tackled by a translation of the origin by an amount $\theta(\varepsilon)$, such that the locations of the sign change of $y^{\prime}$ are fixed (as $y$ is synchronised with the driving force) and independent of $\varepsilon$. (Of course, a certain amount of smoothness is anticipated such that $y^{\prime}$ has the same number of zero's per period as the forcing term). So we introduce

$$
\tilde{\tau}=\Omega \tau-\theta(\varepsilon)
$$

to obtain

$$
\Omega^{2} \frac{\mathrm{d}^{2} y}{\mathrm{~d} \tilde{\tau}^{2}}+\varepsilon \Omega^{2} \frac{\mathrm{d} y}{\mathrm{~d} \tilde{\tau}}\left|\frac{\mathrm{d} y}{\mathrm{~d} \tilde{\tau}}\right|+\varepsilon \Omega r \frac{\mathrm{d} y}{\mathrm{~d} \tilde{\tau}}+y=\varepsilon F_{0} \cos (\tilde{\tau}+\theta)+\varepsilon^{2} h,
$$

where $\theta$ is to be chosen such that $y^{\prime}(\tilde{\tau})=0$ at $\tilde{\tau}=N \pi$. In other words, $\Omega \tau=\omega t=\theta$ corresponds with the phase lag of response $p_{\text {in }}$ to excitation $p_{\text {ex }}$.

When we substitute the following (assumed uniform) asymptotic expansions for $y$ and $\theta$ [21]

$$
y(\tilde{\tau} ; \varepsilon)=y_{0}(\tilde{\tau})+\varepsilon y_{1}(\tilde{\tau})+\varepsilon^{2} y_{2}(\tilde{\tau})+\ldots, \quad \text { and } \quad \theta(\varepsilon)=\theta_{0}+\varepsilon \theta_{1}+\ldots,
$$

and collect like powers of $\varepsilon$, we find for $y_{0}$

$$
\frac{\mathrm{d}^{2} y_{0}}{\mathrm{~d} \tilde{\tau}^{2}}+y_{0}=0, \quad y_{0}^{\prime}(N \pi)=0
$$

This has the general solution

$$
y_{0}(\tilde{\tau})=A_{0} \cos (\tilde{\tau}),
$$

with $A_{0}$ and $\theta_{0}$ to be determined. Although $y_{0}$ is the result of driving force $F$, at this level we don't have any information about their relation yet, so we can't determine the integration constants $A_{0}$ and $\theta_{0}$. Therefore, we continue with the next order $y_{1}$.

$$
\begin{aligned}
\frac{\mathrm{d}^{2} y_{1}}{\mathrm{~d} \tilde{\tau}^{2}}+y_{1}=F_{0} \cos (\tilde{\tau} & \left.+\theta_{0}\right)-2 \Delta \frac{\mathrm{d}^{2} y_{0}}{\mathrm{~d} \tilde{\tau}^{2}}-\frac{\mathrm{d} y_{0}}{\mathrm{~d} \tilde{\tau}}\left|\frac{\mathrm{d} y_{0}}{\mathrm{~d} \tilde{\tau}}\right|-r \frac{\mathrm{d} y_{0}}{\mathrm{~d} \tilde{\tau}} \\
& =F_{0} \cos \left(\tilde{\tau}+\theta_{0}\right)+2 \Delta A_{0} \cos (\tilde{\tau})+A_{0}\left|A_{0}\right| \sin (\tilde{\tau})|\sin (\tilde{\tau})|+r A_{0} \sin (\tilde{\tau})
\end{aligned}
$$

From the arguments that $y$ is the stationary solution and its asymptotic expansion is uniform in $\tilde{\tau}$, it follows that no resonant excitation is allowed in the right hand side of the equation for $y_{1}$. This means that we should suppress the cos- and sin-terms, including the first term of the Fourier expansion of

$$
\sin (\tilde{\tau})|\sin (\tilde{\tau})|=-\frac{1}{\pi} \sum_{n=0}^{\infty} \frac{\sin (2 n+1) \tilde{\tau}}{\left(n^{2}-\frac{1}{4}\right)\left(n+\frac{3}{2}\right)}=\frac{8}{3 \pi} \sin \tilde{\tau}+\ldots
$$

to obtain

$$
F_{0} \cos \theta_{0}=-2 \Delta A_{0}, \quad F_{0} \sin \theta_{0}=\left(\frac{8}{3 \pi}\left|A_{0}\right|+r\right) A_{0}
$$


or

$$
\left[\left(\frac{8}{3 \pi}\left|A_{0}\right|+r\right)^{2}+(2 \Delta)^{2}\right] A_{0}^{2}=F_{0}^{2}, \quad \tan \left(\theta_{0}\right)=-\frac{\frac{8}{3 \pi}\left|A_{0}\right|+r}{2 \Delta} .
$$

In general, the equation for $A_{0}$ has to be solved numerically, from which $\theta_{0}$ follows. There exist two (real) solutions, while if $\left(A_{0}, \theta_{0}\right)$ is a solution, then also $\left(-A_{0}, \theta_{0}+\pi\right)$. So, if convenient, we could assume that $A_{0}$ is positive and maintain $\left|A_{0}\right|=A_{0}$, but this depends on $\theta_{0}$.

The next order $y_{1}$ is then given by

$$
y_{1}(\tilde{\tau})=A_{1} \cos \tilde{\tau}+B_{1} \sin \tilde{\tau}+\frac{1}{4 \pi} A_{0}\left|A_{0}\right| \sum_{n=1}^{\infty} \frac{\sin (2 n+1) \tilde{\tau}}{n(n+1)\left(n^{2}-\frac{1}{4}\right)\left(n+\frac{3}{2}\right)}
$$

with derivative

$$
y_{1}^{\prime}(\tilde{\tau})=-A_{1} \sin \tilde{\tau}+B_{1} \cos \tilde{\tau}+\frac{1}{4 \pi} A_{0}\left|A_{0}\right| \sum_{n=1}^{\infty} \frac{(2 n+1) \cos (2 n+1) \tilde{\tau}}{n(n+1)\left(n^{2}-\frac{1}{4}\right)\left(n+\frac{3}{2}\right)}
$$

and so the boundary condition

$$
\begin{aligned}
& y_{1}^{\prime}(N \pi)=-A_{1} \sin (N \pi)+B_{1} \cos (N \pi)+\frac{1}{4 \pi} A_{0}\left|A_{0}\right| \sum_{n=1}^{\infty} \frac{(2 n+1) \cos ((2 n+1) N \pi)}{n(n+1)\left(n^{2}-\frac{1}{4}\right)\left(n+\frac{3}{2}\right)} \\
& \quad=(-1)^{N} B_{1}+\frac{(-1)^{N}}{4 \pi} A_{0}\left|A_{0}\right| \sum_{n=1}^{\infty} \frac{2 n+1}{n(n+1)\left(n^{2}-\frac{1}{4}\right)\left(n+\frac{3}{2}\right)}=(-1)^{N}\left[B_{1}+\frac{2}{9 \pi} A_{0}\left|A_{0}\right|\right]=0
\end{aligned}
$$

is satisfied by

$$
B_{1}=-\frac{2}{9 \pi} A_{0}\left|A_{0}\right|, \quad \text { because } \quad \sum_{n=1}^{\infty} \frac{2 n+1}{n(n+1)\left(n^{2}-\frac{1}{4}\right)\left(n+\frac{3}{2}\right)}=\frac{8}{9} .
$$

The sum of the telescoping series is easily found by partial fractions and noting the terms cancelling in pairs. Altogether we have

$$
y_{1}(\tilde{\tau})=A_{1} \cos \tilde{\tau}-\frac{2}{9 \pi} A_{0}\left|A_{0}\right| \sin \tilde{\tau}+\frac{1}{4 \pi} A_{0}\left|A_{0}\right| \sum_{n=1}^{\infty} \frac{\sin (2 n+1) \tilde{\tau}}{n(n+1)\left(n^{2}-\frac{1}{4}\right)\left(n+\frac{3}{2}\right)} .
$$

The amplitude $A_{1}$ is to be determined in a similar way as with $y_{0}$ by suppressing resonant terms in $y_{2}$. The next order term $y_{2}$ is obtained from (19) when it is expanded to $O\left(\varepsilon^{2}\right)$ and terms of $O\left(\varepsilon^{2}\right)$ are collected

$$
y_{2}^{\prime \prime}+y_{2}=-\Delta^{2} y_{0}^{\prime \prime}-2 \Delta y_{1}^{\prime \prime}-2 \Delta y_{0}^{\prime}\left|y_{0}^{\prime}\right|-2 y_{1}^{\prime}\left|y_{0}^{\prime}\right|-r y_{1}^{\prime}-r \Delta y_{0}^{\prime}-\theta_{1} F_{0} \sin \left(\tilde{\tau}+\theta_{0}\right)+h
$$

After substituting $y_{0}$ and $y_{1}$, and considering only the terms on the right hand side that are possibly in resonance with the left hand side, we obtain

$$
\begin{aligned}
& y_{2}^{\prime \prime}+y_{2}=\Delta^{2} A_{0} \cos \tilde{\tau}+2 \Delta A_{1} \cos \tilde{\tau}-\frac{4}{9 \pi} \Delta A_{0}\left|A_{0}\right| \sin \tilde{\tau} \\
& +\left(2 \Delta A_{0} \sin \tilde{\tau}+2 A_{1} \sin \tilde{\tau}+\frac{4}{9 \pi} A_{0}\left|A_{0}\right| \cos \tilde{\tau}-\frac{1}{\pi} A_{0}\left|A_{0}\right| \sum_{n=1}^{\infty} \frac{\cos (2 n+1) \tilde{\tau}}{n(n+1)\left(n-\frac{1}{2}\right)\left(n+\frac{3}{2}\right)}\right)\left|A_{0} \sin \tilde{\tau}\right| \\
& \quad+r A_{1} \sin \tilde{\tau}+\frac{2}{9 \pi} r A_{0}\left|A_{0}\right| \cos \tilde{\tau}+r \Delta A_{0} \sin \tilde{\tau}-\theta_{1} F_{0} \cos \theta_{0} \sin \tilde{\tau}-\theta_{1} F_{0} \sin \theta_{0} \cos \tilde{\tau}+\ldots
\end{aligned}
$$

By Fourier expansion it can be found that

$$
\cos \tilde{\tau}|\sin \tilde{\tau}|=\frac{4}{3 \pi} \cos \tilde{\tau}+\ldots, \quad \sum_{n=1}^{\infty} \frac{|\sin \tilde{\tau}| \cos (2 n+1) \tilde{\tau}}{n(n+1)\left(n-\frac{1}{2}\right)\left(n+\frac{3}{2}\right)}=\frac{1}{\pi}\left(\frac{80}{27}-\frac{\pi^{2}}{3}\right) \cos \tilde{\tau}+\ldots
$$


and only higher harmonics otherwise. Suppressing the cos- and sin-terms of (31) thus results into

$$
\begin{aligned}
2 \Delta A_{1}-\theta_{1} F_{0} \sin \theta_{0} & =-\Delta^{2} A_{0}-\left(\frac{1}{3}-\frac{64}{27 \pi^{2}}\right) A_{0}^{3}-\frac{2}{9 \pi} r A_{0}\left|A_{0}\right| \\
\left(\frac{16}{3 \pi}\left|A_{0}\right|+r\right) A_{1}-\theta_{1} F_{0} \cos \theta_{0} & =-\left(\frac{44}{9 \pi}\left|A_{0}\right|+r\right) \Delta A_{0}
\end{aligned}
$$

By solving the linear system (33), we can obtain $A_{1}$ and $\theta_{1}$.

\section{Time-domain Solution}

The solution $y=y_{0}+\varepsilon y_{1}+O\left(\varepsilon^{2}\right)$ ascertains in principle (for small $\varepsilon$ ) a better approximation of $y$ than the leading order approximation $y_{0}$, which would later provide a better approximation of the impedance. We have this full solution as

$$
y(\tilde{\tau} ; \varepsilon)=\left(A_{0}+\varepsilon A_{1}\right) \cos \tilde{\tau}-\frac{2}{9 \pi} \varepsilon A_{0}\left|A_{0}\right| \sin \tilde{\tau}+\frac{1}{4 \pi} \varepsilon A_{0}\left|A_{0}\right| \sum_{n=1}^{\infty} \frac{\sin (2 n+1) \tilde{\tau}}{n(n+1)\left(n^{2}-\frac{1}{4}\right)\left(n+\frac{3}{2}\right)}+\ldots
$$

where $\tilde{\tau}=\omega t-\theta$ and $\theta=\theta_{0}+\varepsilon \theta_{1}+\ldots$ The constants $A_{0}, \theta_{0}$ and $A_{1}, \theta_{1}$ can be determined from (24) and (33) respectively.

Consider first the leading order approximation. Equation (25) for $A_{0}$ has 2 real symmetric solutions (of which we normally need to consider only the positive one), but solving $A_{0}=A_{0}(\Delta)$ is not straightforward. Therefore, it is useful to consider the inverse, $\Delta=\Delta\left(A_{0}\right)$, given by

$$
4 \Delta^{2}=\frac{F_{0}^{2}}{A_{0}^{2}}-\left(\frac{8}{3 \pi}\left|A_{0}\right|+r\right)^{2}
$$

Since $\Delta^{2} \geqslant 0$ we see immediately that solutions exists only for a finite interval in $A_{0}$, while $\Delta \rightarrow \infty$ only when $A_{0} \rightarrow 0$. In particular, we have

$$
A_{0} \simeq \frac{F_{0}}{2|\Delta|}, \quad \tan \theta_{0} \simeq-\frac{r}{2 \Delta} \quad \text { or } \quad \theta_{0} \simeq-\frac{r}{2 \Delta}+n \pi,
$$

which is in exact agreement with the asymptotic behaviour for $\Omega=1+\varepsilon \Delta, \Delta$ large, corresponding to the linear solution (14). In fact, by tracing the solution parametrically as a function of $\Delta$, we can see that if we start with $\theta_{0}=0$ for $\Delta \rightarrow-\infty$, we end with $\theta_{0}=\pi$ for $\Delta \rightarrow \infty$. This way, we have obtained the expression for $A_{0}$ and $\theta_{0}$; see Fig. 2 for an example.
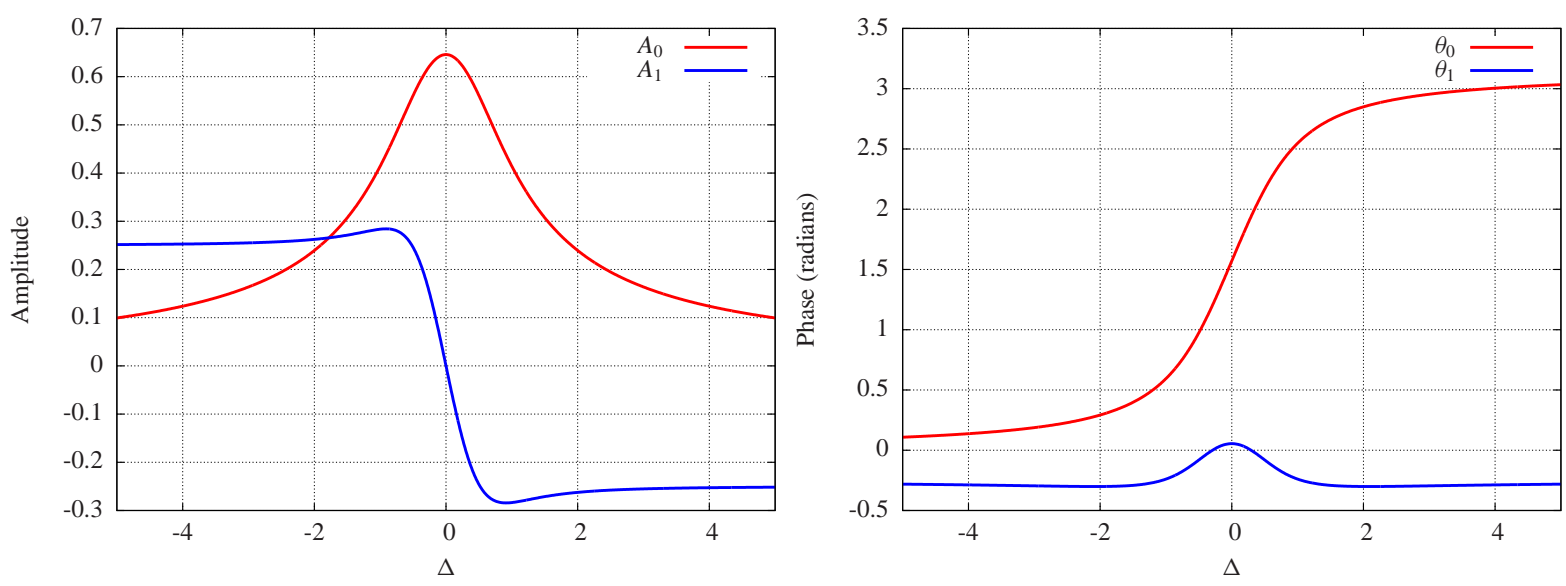

Figure 2. Solution of amplitude $\left(A_{0}, A_{1}\right)$ and phase $\left(\theta_{0}, \theta_{1}\right)$ as a function of $\Delta$, while $r=F_{0}=1$ 
Substituting the obtained value of $A_{0}$ and $\theta_{0}$ in (33), we can solve the linear algebraic system to obtain $A_{1}$ and $\theta_{1}$. This way, we have determined all the coefficients in (34); hence, the solution $y$ is known which, when used with $(8)$, gives $p_{\text {in }}$

$$
\begin{aligned}
p_{\text {in }}=2 \varepsilon \rho_{0} c_{0}^{2} & \frac{\ell S_{n}}{V}\left[\left(A_{0}+\varepsilon A_{1}\right) \cos (\omega t-\theta)\right. \\
& \left.-\frac{2}{9 \pi} \varepsilon A_{0}\left|A_{0}\right| \sin (\omega t-\theta)+\frac{1}{4 \pi} \varepsilon A_{0}\left|A_{0}\right| \sum_{n=1}^{\infty} \frac{\sin (2 n+1)(\omega t-\theta)}{n(n+1)\left(n^{2}-\frac{1}{4}\right)\left(n+\frac{3}{2}\right)}+\ldots\right] .
\end{aligned}
$$

From this solution and (6) we may determine the neck velocity $u_{n}$

$$
\begin{aligned}
u_{n}=2 \varepsilon \omega \ell & {\left[\left(A_{0}+\varepsilon A_{1}\right) \sin (\omega t-\theta)\right.} \\
& \left.+\frac{2}{9 \pi} \varepsilon A_{0}\left|A_{0}\right| \cos (\omega t-\theta)-\frac{1}{4 \pi} \varepsilon A_{0}\left|A_{0}\right| \sum_{n=1}^{\infty} \frac{(2 n+1) \cos (2 n+1)(\omega t-\theta)}{n(n+1)\left(n^{2}-\frac{1}{4}\right)\left(n+\frac{3}{2}\right)}+\ldots\right],
\end{aligned}
$$

which will be used to obtain the impedance of the resonator in a later section.

\section{IV.A. Comparison in Time-domain with a Fully Numerical Solution}

The solution (34), correct till $O(\varepsilon)\left(y_{0}\right)$ and $O\left(\varepsilon^{2}\right)\left(y_{0}+\varepsilon y_{1}\right)$, are compared with a fully numerical solution of (10), obtained by Mathematica with a standard Runge-Kutta routine, see Fig. 3, In both cases $r=0.2, \Delta=1$, while $\varepsilon=0.28$ in the left figure and $\varepsilon=0.88$ in the right. Note that this last case is added to see how the solution behaves for values of $\varepsilon$ that are really not small anymore. The one with $\varepsilon=0.28$ is indeed remarkably accurate for $y_{0}+\varepsilon y_{1}$, and we may observe an error of $y_{0}$ and $y_{0}+\varepsilon y_{1}$ compared to $y$ that follows indeed the predicted behaviour of $O(\varepsilon)$ and $O\left(\varepsilon^{2}\right)$. The one with $\varepsilon=0.88$ cannot be expected to be really accurate, but surprisingly the results are still of the right order of magnitude.
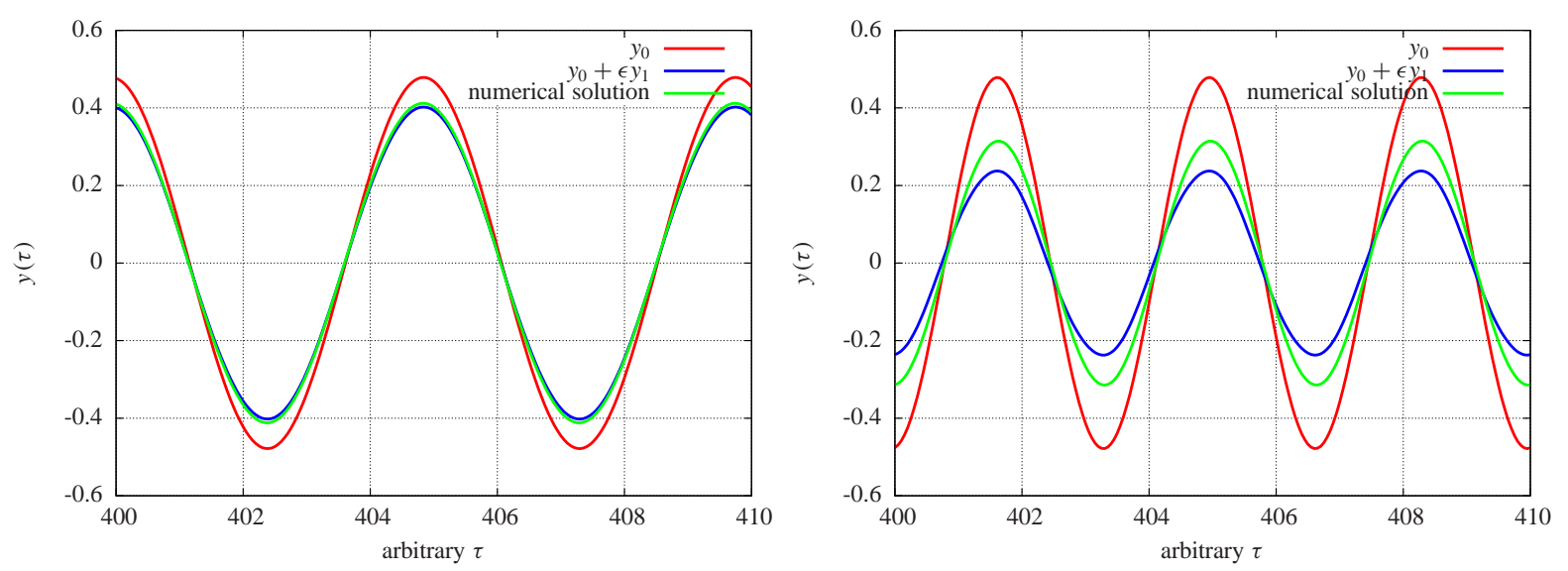

Figure 3. Comparison of solution $y_{0}$ and $y_{0}+\varepsilon y_{1}$ for $r=0.2, \Delta=1$ and $\varepsilon=0.28$ (left) and $\varepsilon=0.88$ (right) with a fully numerical solution.

We note, however, that there is always the assumption that $\Delta=O(1)$ and $1-\Omega^{2}=O(\varepsilon)$. In other words, the validity of the resonance solution is for an interval in frequency of $\omega=\omega_{0}(1+O(\varepsilon))$. When we leave this interval, the non-resonant solution (17) should gradually become applicable.

\section{Impedance Calculation}

In order to obtain realistic numbers, we will consider the impedance $Z$ as the effective impedance of an array of Helmholtz resonators, where the spatially averaged neck velocity is identified to the external 
acoustic velocity. Therefore, we add a porosity factor $S_{n} / S_{b}$ to $u_{n}$ and obtain

$$
v_{\mathrm{ex}}=\frac{S_{n}}{S_{b}} u_{n} .
$$

Then we have to define what we mean with impedance for a sound field that is not entirely harmonic anymore. The natural choice is to define the impedance as the ratio of the Fourier transforms of the external pressure $p_{\text {ex }}$ and (minus) the external velocity $v_{\mathrm{ex}}$ at excitation frequency $\omega$. Taking the Fourier transforms of $p_{\mathrm{ex}}$ and $v_{\mathrm{ex}}$, we have for $\eta>0$

$$
\hat{p}_{\text {ex }}(\eta)=\frac{1}{2 \pi} \int_{-\infty}^{\infty} p_{\text {ex }}(t) \mathrm{e}^{-\mathrm{i} \eta t} \mathrm{~d} t=\varepsilon^{2} \rho_{0} c_{0}^{2} \frac{\ell S_{n}}{V} F_{0} \delta(\eta-\omega)
$$

and

$$
\begin{aligned}
\hat{v}_{\mathrm{ex}}(\eta)=\frac{1}{2 \pi} \int_{-\infty}^{\infty} v_{\mathrm{ex}}(t) \mathrm{e}^{-\mathrm{i} \eta t} \mathrm{~d} t=\frac{S_{n}}{S_{b}} \varepsilon \omega \ell \mathrm{e}^{-\mathrm{i} \theta}\left[-\mathrm{i}\left(A_{0}+\varepsilon A_{1}\right) \delta(\eta-\omega)\right. \\
\left.\quad+\frac{2}{9 \pi} \varepsilon A_{0}\left|A_{0}\right| \delta(\eta-\omega)-\frac{1}{4 \pi} \varepsilon A_{0}\left|A_{0}\right| \sum_{n=1}^{\infty} \frac{(2 n+1) \delta(\eta-(2 n+1) \omega)}{n(n+1)\left(n^{2}-\frac{1}{4}\right)\left(n+\frac{3}{2}\right)}+\ldots\right],
\end{aligned}
$$

and so (with $V=L S_{b}$ ) we obtain

$$
Z(\omega)=-\frac{\hat{p}_{\mathrm{ex}}(\omega)}{\hat{v}_{\mathrm{ex}}(\omega)}=\frac{\varepsilon \rho_{0} c_{0}^{2} F_{0}}{L \omega} \cdot \frac{-\mathrm{i} \mathrm{e}^{\mathrm{i} \theta}}{A_{0}+\varepsilon A_{1}+\mathrm{i} \frac{2}{9 \pi} \varepsilon A_{0}\left|A_{0}\right|} .
$$

It is interesting to consider $Z$ to leading order in $\varepsilon$

$$
Z(\omega) \simeq \frac{\varepsilon \rho_{0} c_{0}^{2} F_{0}}{L \omega} \frac{-\mathrm{i} \mathrm{e}^{\mathrm{i} \theta_{0}}}{A_{0}}=\rho_{0} c_{0} \frac{c_{0}}{\omega L}\left(\frac{R}{\rho_{0} \omega_{0} \ell}+\frac{4}{3 \pi} \frac{\left\|u_{n}\right\|}{\omega \ell}+2 \mathrm{i} \frac{\omega-\omega_{0}}{\omega_{0}}\right)
$$

(where $\left\|u_{n}\right\|$ denotes the amplitude of $u_{n}$ ) and observe that indeed $\operatorname{Re}(Z)$ is of the often assumed form $a+b\left\|u_{n}\right\|$. Although our $a$ and $b$ are no constants and depend on $\omega$, this is a higher order effect because $\omega=\omega_{0}(1+O(\varepsilon))$. To leading order they are constant. $\operatorname{Im}(Z)$ is independent of the excitation amplitude. In order to illustrate formula (42), we have plotted in Fig. 4 resistance $\operatorname{Re}(Z)$ and reactance $\operatorname{Im}(Z)$ as a function of $\Omega$, obtained for a typical geometry at different driving amplitudes, corresponding with $\varepsilon$ varying from 0.05 to 0.28 . As may be expected from (43), the main effect of the forcing amplitude is in the resistance. The reactance is practically independent of it. Typically, the resistance, being highest at or near the resonance frequency and decaying along both sides, increases everywhere with the amplitude, but more for frequencies less than resonance.

This behaviour may be compared in Fig. 5 with the measurements and predictions given by Motsinger and Kraft in [11]. Their predictions are (a.o.) based on a resistance of the form $R=\rho_{0} c_{0}(a+b|v|)$ with suitably chosen $a$ and $b$. Unfortunately, only little experimental data for the higher amplitudes are available. The parameter values we used are based on $\omega_{0} / 2 \pi=2200 \mathrm{~Hz}, \ell=0.001 \mathrm{~m}, L=0.035 \mathrm{~m}$, $S_{n} / S_{b}=0.05, r=0.1$, and $\varepsilon$-values for $125 \mathrm{~dB}$ corresponding with $\varepsilon=0.3125,130 \mathrm{~dB}$ with $\varepsilon=$ $0.4168,135 \mathrm{~dB}$ with $\varepsilon=0.5558,140 \mathrm{~dB}$ with $\varepsilon=0.7411$ and $145 \mathrm{~dB}$ with $\varepsilon=0.9883$.

The agreement is reasonable, taking into account that the $\varepsilon$ 's are not very small and no experimental data are available in this frequency range for the higher amplitudes. Especially the increase of the maximum with the amplitude is confirmed. Only for the highest amplitude (with a value of $\varepsilon=0.99$ that is far beyond what could be considered asymptotically "small") and frequencies well above resonance the decay predicted by [11] is not confirmed.

\footnotetext{
${ }^{3}$ For $\omega$ well away from resonance, we may obtain from (17) in a similar way the usual

$$
Z(\omega) \simeq \frac{S_{b}}{S_{n}}\left(R+\mathrm{i} \rho_{0} \ell \omega_{0}\left(\frac{\omega}{\omega_{0}}-\frac{\omega_{0}}{\omega}\right)\right) .
$$
}

Being void of nonlinear effects, this case will not be considered here further. 

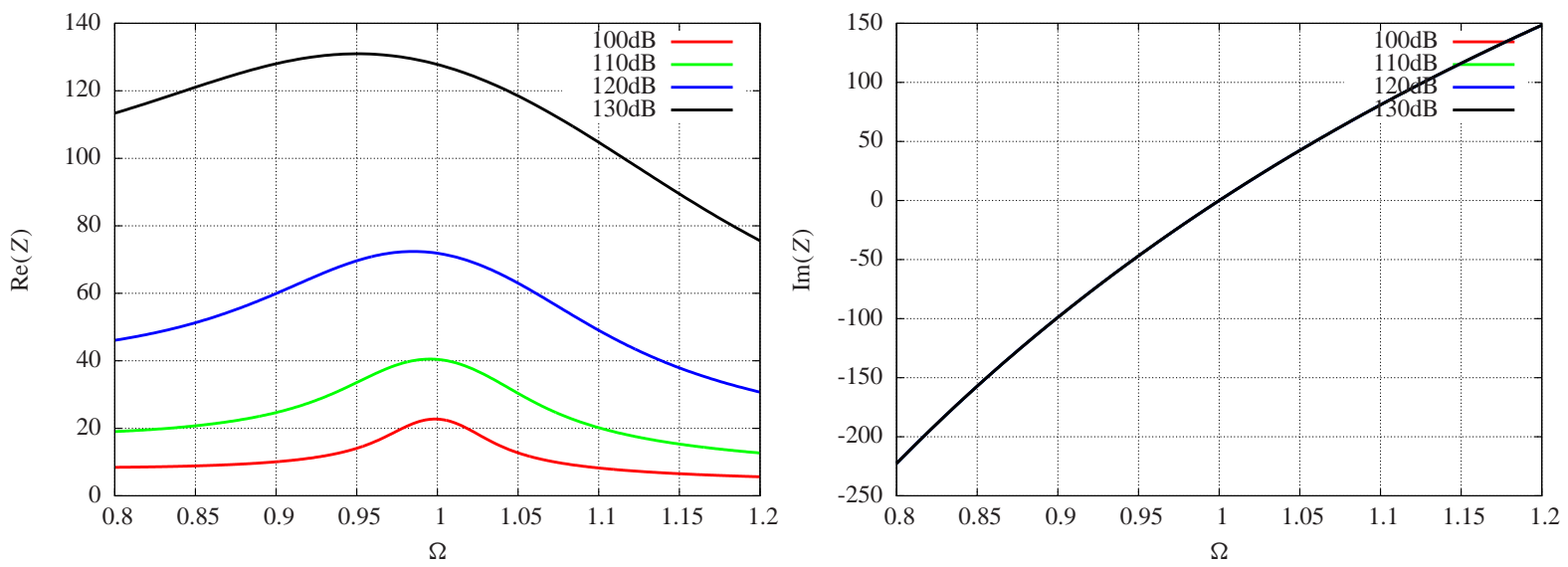

Figure 4. Real and imaginary parts of impedance $Z$ for a Helmholtz resonator as a function of nondimensional frequency at different driving amplitudes. The realistic configuration that is chosen corresponds with $S_{n} / S_{b}=0.05, r=0.2, \omega_{0}=9086.9 \mathrm{rad} / \mathbf{s}, L=0.035 \mathrm{~m}$, $\ell=0.002 \mathbf{m} .100 \mathrm{~dB}$ corresponds with $\varepsilon=0.0497,110 \mathrm{~dB}$ with $\varepsilon=0.0884,120 \mathrm{~dB}$ with $\varepsilon=0.1572$, and $130 \mathrm{~dB}$ with $\varepsilon=0.2796$.

\section{V.A. Comparison of Impedances Based on $y_{0}$ and $y_{0}+\varepsilon y_{1}$ Approximations}

It is of interest to know when the driving amplitude becomes large enough to warrant the extra term $\varepsilon y_{1}$ in the approximation of $Z$. Shown in Fig. 6 is the comparison of the impedance values obtained from $y_{0}$ and $y_{0}+\varepsilon y_{1}$ approximations for different values of $\varepsilon$. Taking the same realistic geometry as above (Fig. 4), the value of $\varepsilon$ vary from $\sim 0.05$ to 0.28 as the external driving amplitude is changed from $100 \mathrm{~dB}$ to $130 \mathrm{~dB}$. We see that $O(\varepsilon)$ correction in the resulting resistance (the reactance is practically independent, especially near resonance) can be neglected for the lower amplitudes, but is indeed essential for the higher amplitudes.

\section{Conclusions}

A systematic approximation of the hydrodynamically non-linear Helmholtz resonator equation is obtained, including the resulting impedance if the resonator is applied in an acoustic liner. To leading order, the usually assumed form of the resistance, $a+b|v|$, is recovered.

Our approach, based on systematic use of asymptotic analysis, allows higher order corrections, which indeed are shown to be important and relevant for practical configurations involving high amplitudes.

The real part of the found impedance (the resistance) shows the usual characteristic behaviour as a function of frequency, namely a maximum at or near the resonance frequency and a decay along both sides. All values increase with the amplitude, but slightly more for the frequencies less than resonance.
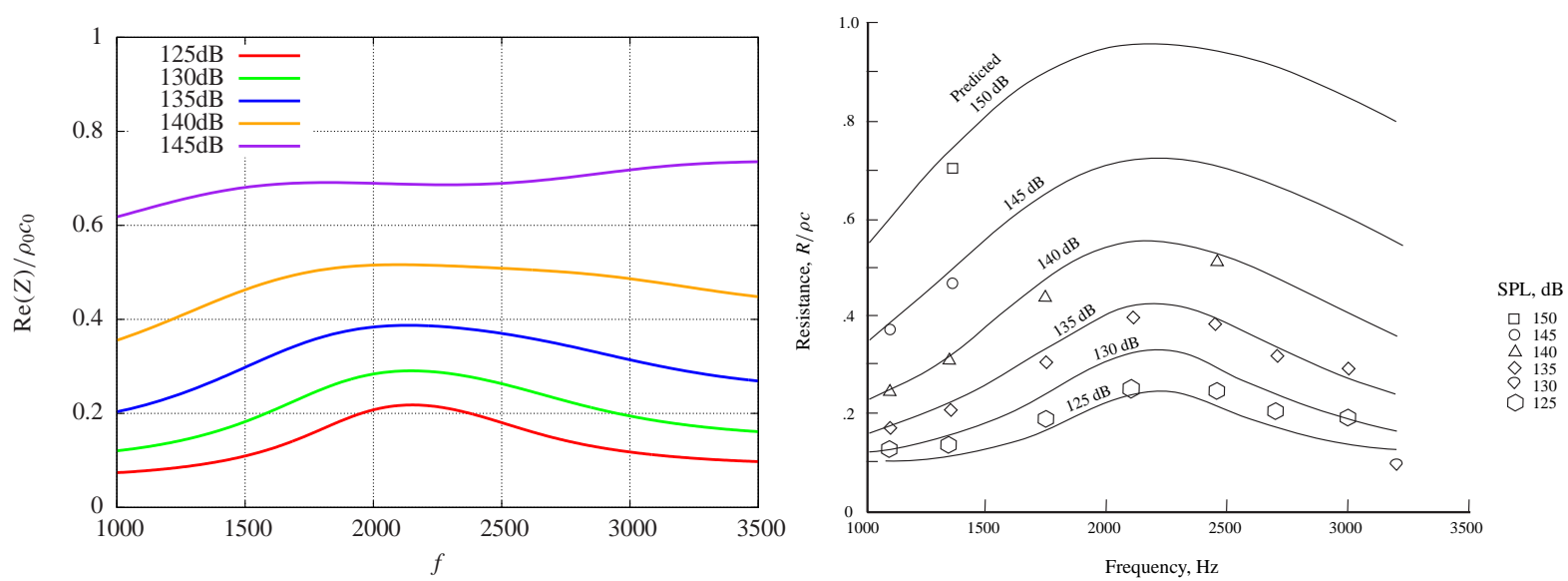

Figure 5. Comparison of (42) with measurements and predictions of $\operatorname{Re}(Z) / \rho_{0} c_{0}$ given in [11]. Estimated values of $\varepsilon$ vary from 0.31 to 0.99 . 

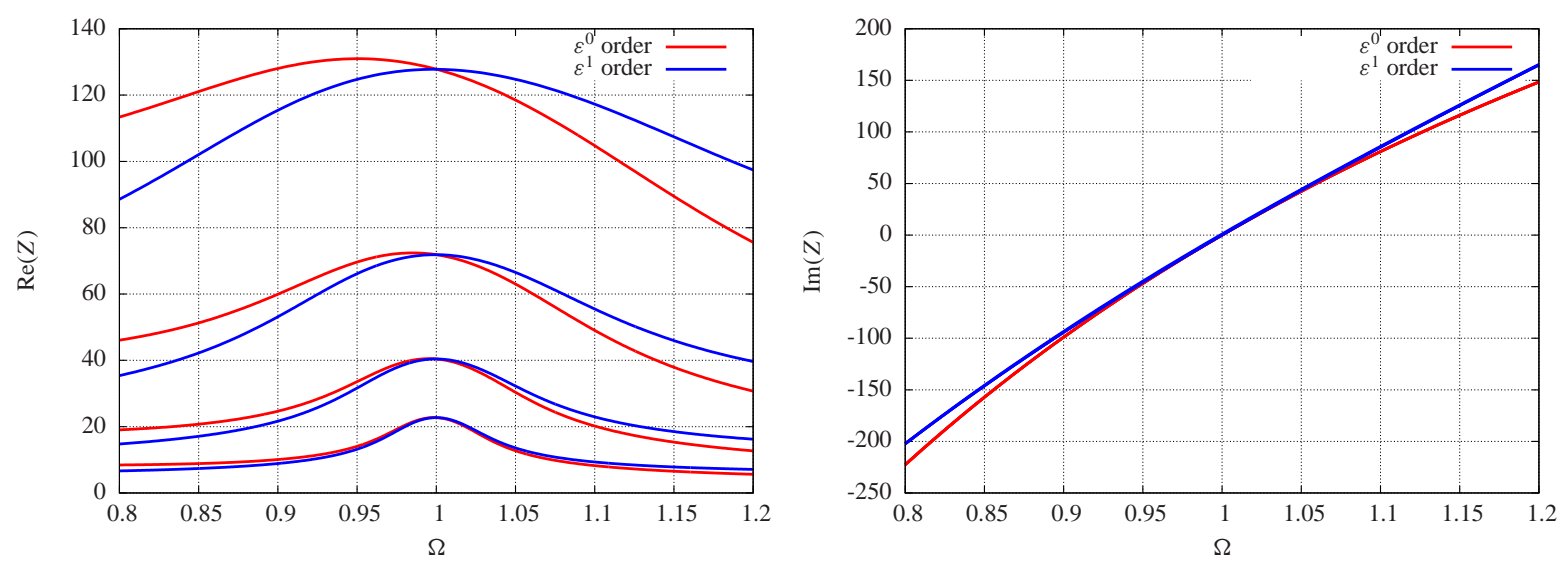

Figure 6. Comparison of the impedance obtained from the $y_{0}$ and $y_{0}+\varepsilon y_{1}$ approximations for $100 \mathrm{~dB}, 110 \mathrm{~dB}, 120 \mathrm{~dB}$ and $130 \mathrm{~dB}$. Same configuration as in Fig. 4

The imaginary part of the impedance (the reactance) is linear in frequency in a way that it vanishes at resonance and is practically independent of the amplitude.

\section{Acknowledgements}

We gratefully acknowledge the support from the European Union through ITN-project FlowAirS (contract no. FP7-PEOPLE-2011-ITN-289352), with coordinator Yves Aurégan.

\section{Appendix. Stability of Stationary Solution}

From the physical origin of the problem, it is very likely that there exists a stable steady solution for a steady external forcing, such that we are not approximating a solution that just would not exist in any realisation. We have checked this mathematically by proving the boundedness of a small perturbation $\xi$ of our solution $y$ in (10), satisfying the following equation

$$
\left(y^{\prime \prime}+\xi^{\prime \prime}\right)+\varepsilon\left(y^{\prime}+\xi^{\prime}\right)\left|y^{\prime}+\xi^{\prime}\right|+\varepsilon r\left(y^{\prime}+\xi^{\prime}\right)+(y+\xi)=\varepsilon F(\tau) .
$$

Since, by assumption, $y$ is any solution of the original equation, we have to linear order for small $\xi$ (and a slight error near the zero's of $y^{\prime}+\xi^{\prime}$ )

$$
\xi^{\prime \prime}+\phi(\tau) \xi^{\prime}+\xi=0
$$
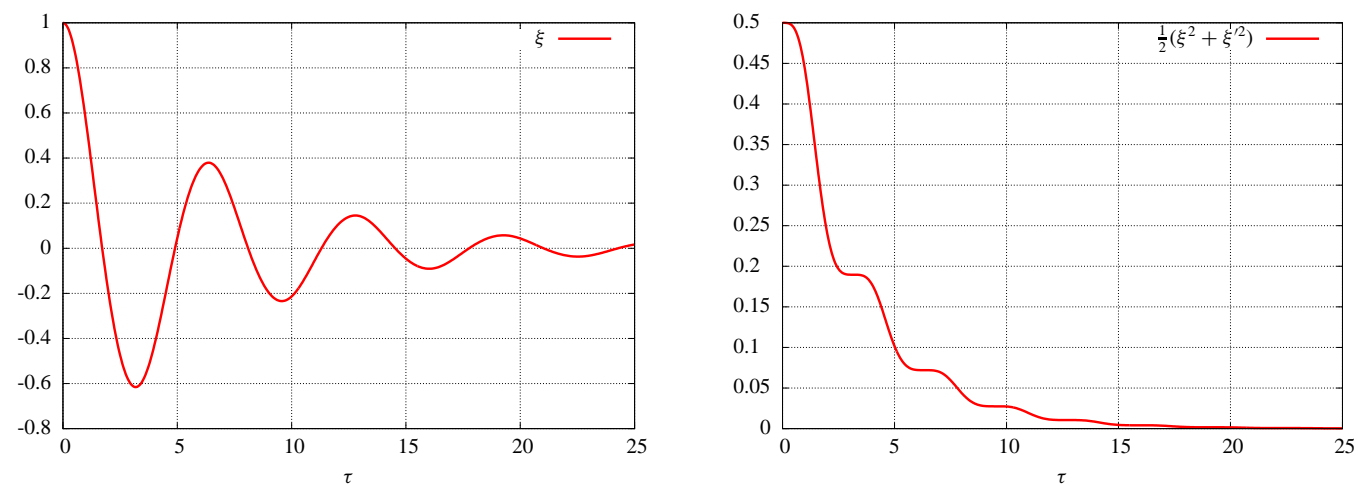

Figure 7. Amplitude $\xi$ and mechanical energy $\frac{1}{2}\left(\xi^{2}+\xi^{\prime 2}\right)$ of a superimposed perturbation when it starts from $\xi(0)=1 \quad \xi^{\prime}(0)=0$. The full solution was approximated by $y=A_{0} \cos (\Omega \tau)$ with $\varepsilon=0.16, \Omega=1$ and $r=0.2$. 
where $\phi(\tau)=\varepsilon\left(r+2\left|y^{\prime}\right|\right) \geqslant 0$ (even strictly positive if $r>0$ ). We assume an initial condition with $\xi(0)^{2}+\xi^{\prime}(0)^{2}=E_{0}^{2}$. From (45) we have for the mechanical energy $\frac{1}{2}\left(\xi^{2}+\xi^{\prime 2}\right)$

$$
\frac{\mathrm{d}}{\mathrm{d} \tau}\left(\frac{1}{2}\left(\xi^{2}+\xi^{\prime 2}\right)\right)=\xi^{\prime} \xi^{\prime \prime}+\xi^{\prime} \xi=-\phi \xi^{\prime 2} \leqslant-\phi\left(\xi^{2}+\xi^{\prime 2}\right)
$$

It follows that $\frac{\mathrm{d}}{\mathrm{d} \tau} \ln \left(\xi^{2}+\xi^{\prime 2}\right) \leqslant-2 \phi$. After integration and using the positivity of $\phi$ we find eventually

$$
\xi^{2}+\xi^{2} \leqslant E_{0}^{2} \exp \left(-2 \int_{0}^{\tau} \phi(\tau) \mathrm{d} \tau\right) .
$$

Hence it follows that perturbations are bounded and will decay to zero, confirming the existence of a stable stationary solution. Shown in Fig. 7 is a plot of $\xi$ and $\frac{1}{2}\left(\xi^{2}+\xi^{\prime 2}\right)$.

\section{References}

${ }^{1}$ C. Bréard, A. Sayma, M. Imregun, A. G. Wilson, and B. J. Tester, "A CFD-based non-linear model for the prediction of tone noise in lined ducts," 7th AIAA/CEAS Aeroacoustics Conference, 2001, AIAA-2001-2176.

${ }^{2}$ A. McAlpine, M. J. Fisher, and B. J. Tester, “"Buzz-saw" Noise: A Comparison of Modal Measurements with an Improved Prediction Method," Journal of Sound and Vibration, Vol. 306, No. 3-5, 2007, pp. 419-443.

${ }^{3}$ U. Ingard and H. Ising, "Acoustic Nonlinearity of an Orifice," The Journal of the Acoustical Society of America, Vol. 42, No. 1, 1967, pp. 6-17.

${ }^{4}$ B. T. Zinn, "A Theoretical Study of Non-Linear Damping by Helmholtz Resonators," Journal of Sound and Vibration, Vol. 3, 1970, pp. 347-356.

5 J. H. M. Disselhorst and L. van Wijngaarden, "Flow in the Exit of Open Pipes during Acoustic Resonance," Journal of Fluid Mechanics, Vol. 99, No. 2, 1980, pp. 293-319.

${ }^{6}$ A. Cummings and W. Eversman, "High Amplitude Acoustic Transmission through Duct Terminations: Theory," Journal of Sound and Vibration, Vol. 91, No. 4, 1983, pp. 503-518.

${ }^{7}$ S. W. Rienstra and A. Hirschberg, "An Introduction To Acoustics," Tech. rep., Technische Universiteit Eindhoven, 2012, revised and updated version of IWDE 92-06, http: //www.win.tue.nl/ s joerdr/papers/boek.pdf.

${ }^{8}$ U. Ingard and S. Labate, "Acoustic Circulation Effects and the Nonlinear Impedance of Orifices," Journal of Acoustic Society of America, Vol. 22, 1950, pp. 211-219.

${ }^{9}$ T. H. Melling, "The Acoustic Impendance of Perforates at Medium and High Sound Pressure Levels," Journal of Sound and Vibration, Vol. 29, No. 1, 1973, pp. $1-65$.

${ }^{10}$ A. W. Guess, "Calculation of perforated plate liner parameters from specified acoustic resistance and reactance," Journal of Sound and Vibration, Vol. 40, No. 1, 1975, pp. 119 - 137.

${ }^{11}$ H. H. Hubbard and Acoustical Society of America, Aeroacoustics of Flight Vehicles: Noise sources, Aeroacoustics of Flight Vehicles: Theory and Practice. Volume 1 Noise Sources; Volume 2 Noise Control, Published for the Acoustical Society of America through the American Institute of Physics, 1991.

12 A. S. Hersh, B. E. Walker, and J. W. Celano, "Helmholtz Resonator Impedance Model, Part 1: Nonlinear Behavior," AIAA Journal, Vol. 41, No. 5, 2003, pp. 795-808.

${ }^{13}$ C. K. W. Tam and K. A. Kurbatskii, "Microfluid Dynamics and Acoustics of Resonant Liners," AIAA Journal, Vol. 38, No. 8, 2000, pp. 1331-1339.

${ }^{14}$ C. K. W. Tam, K. A. Kurbatskii, K. K. Ahuja, and Jr. R. J. Gaeta, "A Numerical and Experimental Investigation of the Dissipation Mechanisms of Resonant Acoustic Liners," Journal of Sound and Vibration, Vol. 245, No. 3, 2001, pp. 545-557.

${ }^{15}$ C. K. W. Tam, H. Ju, M. G. Jones, and T. L. Parrott, “A computational and experimental study of slit resonators,” Journal of Sound and Vibration, Vol. 284, 2005, pp. 947-984.

${ }^{16}$ J. M. Roche, L. Leylekian, G. Delattre, and F. Vuillot, "Aircraft Fan Noise Absorption: DNS of the Acoustic Dissipation of Resonant Liners," 15th AIAA/CEAS Aeroacoustics Conference, 2009, AIAA Paper 2009-3146.

${ }^{17}$ C. K. W. Tam, H. Ju, M. G. Jones, W. R. Watson, and T. L. Parrott, "A Computational and Experimental Study of Resonators in Three Dimensions," 15th AIAA/CEAS Aeroacoustics Conference, AIAA Paper 2009-3173.

${ }^{18} \mathrm{Q}$. Zhang and D. J. Bodony, "Numerical Simulation of Two-Dimensional Acoustic Liners with High Speed Grazing Flow," AIAA Journal, Vol. 49, No. 2, pp. 365-382.

${ }^{19}$ D. Innes and D. G. Crighton, "On a Non-Linear Differential Equation Modelling Helmholtz Resonator Response," Journal of Sound and Vibration, Vol. 131, No. 2, 1989, pp. 323-330.

${ }^{20}$ R. M. M. Mattheij, S. W. Rienstra, and J. H. M. ten Thije Boonkkamp, Partial Differential Equations: Modeling, Analysis, Computation, Society for Industrial and Applied Mathematics, 2005.

${ }^{21}$ R. E. Mickens, An Introduction to Nonlinear Oscillations, Cambridge University Press, 1981. 